\title{
Validation of the WHOQOL-Bref instrument in Brazilian sign language (Libras)
}

\author{
Soraya B. R. Duarte ${ }^{1,2}$ - Neuma Chaveiro ${ }^{1,3}$. Adriana R. de Freitas ${ }^{1,4}$ - Maria Alves Barbosa' ${ }^{1}$ Suzi Camey ${ }^{5}$. \\ Marcelo P. Fleck ${ }^{6} \cdot$ Celmo C. Porto $^{1} \cdot$ Cássio L. Rodrigues $^{7}{ }^{1} \cdot$ Dolors Rodríguez-Martín $^{8}$
}

Accepted: 11 August 2020

(c) Springer Nature Switzerland AG 2020

\begin{abstract}
Purpose The recognition of the Brazilian Sign Language (Libras) as the official language of the Brazilian deaf, in 2002, reaffirms the linguistic and cultural particularities of the deaf population. Therefore, there is a lack of a validated instrument for assessing the Quality of Life of deaf people using Libras. With authorization from the World Health Organization (WHO), a version of the WHOQOL-Bref in Libras was developed, called WHOQOL-Bref/Libras. However, its psychometric properties have not been examined as yet. Therefore, the purpose of this work is to perform the psychometric validation of the WHOQOL-Bref/Libras.

Methods WHOQOL-Bref/Libras and a sociodemographic questionnaire were applied to 311 deaf people from the five Brazilian regions. To assess temporal stability, the questionnaire was readministered to 52 deaf people, over an interval of 2 weeks. Results WHOQOL-Bref/Libras demonstrated satisfactory psychometric values for reliability, discriminant and construct validity, temporal stability, and internal consistency. Cronbach's alpha coefficient showed satisfactory values for each of the WHOQOL-Bref domains: Physical health (0.641), Psychological (0.705), Environment (0.710), and Overall-Bref domains (0.873). The WHOQOL-Bref/Libras is the appropriate option to assess the quality of life of deaf people who communicate through Libras.

Conclusion WHOQOL-Bref/Libras had a satisfactory psychometric performance; therefore, it is a valid option that will provide autonomous participation for the deaf in quality of life investigations.
\end{abstract}

Keywords World Health Organization · Quality of life · Deaf · Brazilian sign language · Validation

\section{Introduction}

Building a fair society requires respect for diversity and the right to equality. In the case of the deaf, it is mandatory to recognize and value sign language and the culture of the deaf population. Before becoming official, sign language was named in several ways: language of gestures, language

Soraya B. R. Duarte

sorayaduarte@ifg.edu.br

1 Programa de Pós Graduação em Ciências da Saúde Faculdade de Medicina, Universidade Federal de Goiás, Goiânia, Brazil

2 Instituto Federal de Educação Ciência e Tecnologia de Goiás, Goiânia, Brazil

3 Faculdade de Letras, Universidade Federal de Goiás, Goiânia, Brazil

4 Pontifícia Universidade Católica de Goiás, Goiânia, Brazil of the deaf, gestures, mime, pantomime or hand movements. In general, the comprehension of deaf culture was influenced by the oralist conception that argues that the deaf should speak and be oralized at any cost.

The social relationships provided by the deaf community allow the deaf to build a representation of themselves in the world. This justifies the result of research that shows that

5 Departamento de Estatística, Instituto de Matemática, Universidade Federal do Rio Grande do Sul, Porto Alegre, Brasil

6 Departamento de Psiquiatria e Medicina Legal, Faculdade de Medicina, Universidade Federal do Rio Grande do Sul, Porto Alegre, Brazil

7 Instituto de Informática, Universidade Federal de Goiás, Goiânia, Brazil

8 Departament d'Infermeria Fonamental i Medicoquirúrgica de l'Escola d'Infermeria de la Facultad de Medicina i Ciències de la Salut de la Universitat de Barcelona, Barcelona, Spain 
deaf people who use sign language can have similar quality of life scores for social relationships with the hearing population, but the hard of hearing can have significantly worse social relationships than the other two groups [1]. Thus, a characteristic that stands out in these social relationships is sign language. In addition to being a linguistic system, a sign language is an element of constitution of the deaf subject, adding their identity and culture. Therefore, deafness is not a disability, but a difference in experiencing the world [2-5]. In Brazil, the Brazilian Sign Language (Libras) was recognized by law as a means of communication and expression for the deaf community in 2002 [5]. The right of the deaf to communicate in their natural language, i.e., in Libras, is legally guaranteed in Brazil, without the imposition of speaking the main language of the country.

The inclusion of the deaf in health services is characterized by the difficulty of communication, constituting one of the major obstacles to health care, capable of impairing care and compromising the quality of care and health of deaf people [6-10]. Pendergrass [11] et al. state that when Health Care Providers (HCPs) are unaware that there is a cultural view of deafness, significant barriers may exist between the HCP and the deaf individual. Furthermore, knowing the health indicators of the most different populations has become a recent and growing priority [12]. In order to find quality of life measures, it was necessary to create assessment instruments. Currently, there are different instruments, but not all are available and validated in Libras, which precludes objective, reliable, clear and precise assessment of the influence of deafness in the lives of deaf people.

To measure the quality of life of the most diverse population groups, WHO has created the World Health Organization Quality of Life (WHOQOL Group), which recommends a rigorous methodology for the translation and validation of their instruments for different languages. The World Health Quality of Life Instrument contains, in its abbreviated version (WHOQOL-Bref), four domains, 24 facets and 26 questions, two of which are general questions. This instrument is now available in over forty languages [13]. The WHOQOLBref instrument was selected because it is an instrument that adopts the definition of health as a complete state of physical, mental and social well-being and not simply the absence of disease. In addition, the instrument was created using a multicentric approach with a cross-cultural focus that allows its application and validation in different cultures, including deaf people. WHOQOL-Bref allows comparisons of data between populations, according to the International Classification of Functioning, Disability and Health [14].

WHOQOL-Bref has been used with deaf people in other countries in different languages, and this administration for its use varies [15-17]. These studies indicate that the sign language version of WHOQOL-Bref can be used reliably to assess the quality of life of deaf people. Chaveiro et al. [18,
19] constructed a version of the WHOQOL-Bref instrument in Brazilian sign language to evaluate the Brazilian deaf population's quality of life. The translation and construction of the WHOQOL-Bref/Libras were authorized by WHO. The methodology proposed by the World Health Organization was used to construct instruments adapted to the deaf community using Libras. However, the investigation of the psychometric properties of WHOQOL-Bref/Libras was not the objective of these studies.

The aim of this paper is to evaluate the psychometric properties of WHOQOL-Bref in the Brazilian Sign Language version (WHOQOL-Bref/Libras).

\section{Methods}

\section{Field test}

The field test was carried out in three steps. In each of them, there was the distribution of user code and specific passwords, in order to facilitate access to the instrument and facilitate the organization of the databases. The steps were: step (1) conducting the pilot test to verify the usability of the software and methodological procedures of the application; step (2) Field test with local respondents in the computer lab and geographically dispersed over the internet, and step (3) Field retest with local respondents in the computer lab. Fifteen deaf collaborators were selected, who received eight hours of training on the WHOQOL-Libras project, the WHOQOL-Bref/Libras research, how to use the software and guidelines to assist in capturing participants for the study.

\section{Type and place of study}

Data for the WHOQOL-Bref/Libras field test were collected using a cross-sectional, descriptive, exploratory and quantitative design. The application of WHOQOL-Bref/Libras was performed in a virtual space where the software of this instrument is hosted. The means used to interact with the deaf was the WHOQOL-Libras Group's email address and social networks, through which new participants emerged, who were contacted through video invitations, containing the web link to the tool, the user code and password to participate in the survey. All respondents received information about the objectives of the study and about the confidentiality of the information.

\section{Population and sample}

Sample formation was for convenience. Deaf associations, educational and religious institutions from the five Brazilian 
regions were contacted. The five regions of Brazil are: North, Northeast, Midwest, Southeast, and South.

Knowing that the most effective way to contact the deaf is through social networks, videos were made about the research objectives, the WHOQOL-Libras Project and video invitations were used to communicate with participants. The scripts of the videos were performed by deaf translators from the Goiânia Deaf Association. The language used to disseminate, inform, explain and invite was Libras.

The deaf were recruited according to the following inclusion criteria: (a) age between 18 and 65 years, and (b) communication by Libras. Participants were stratified by sex, age, income, education, marital status, number of children, work status and subjective perception of health status.

\section{Data collection}

Two instruments that make up the WHOQOL-Bref/Libras software were used to remotely collect data from the field test: the sociodemographic questionnaire and the WHOQOL-Bref/Libras instrument, composed of 26 questions, with 2 questions referring to the general aspects of quality of life and 24 to specific aspects [19]. The two general questions are: "How would you rate your quality of life?" And "How satisfied are you with your health?" The 24 questions of specific aspects cover four domains, according to the Table 1. Responses were given on a Likert scale, designed and tested to reflect intensity, capacity, frequency and evaluation. Domain scores are scaled in a positive direction, with higher scores denoting greater quality of life [13].

The WHOQOL-Libras software consists of (1) presentation, encompassing the concept of WHO quality of life and the "Quality of Life" sign created in Libras; (2) instruction with explanation and guidance for use of the software; (3) access to the questionnaires, Informed Consent and option "I read and agree," question about the participant's perception of their health status, sociodemographic questionnaire, WHOQOL-Bref/Libras; (4) Contact Us option (name, email, message and "send message"); and (5) Exit. The instruments are self-explanatory and self-administered. The collected data were recorded in coded form, in a database. Data collection lasted 3 months.

In order to assess the temporal stability of the instrument, two sets of data were collected: the first set included the deaf who answered WHOQOL-Bref Libras only once; the second set included those who answered the questionnaire twice to perform a retest. To participate in the retest, the following criteria had to be satisfied: the participant should have filled out the questionnaire in the field test, in a computer laboratory previously determined by the WHOQOL-Bref/Libras
Table 1 WHOQOL-BREF domains [13]

\begin{tabular}{ll}
\hline Domain & Questions and Facets incorporated within domains \\
\hline 1. Physical health & 1. Pain and discomfort \\
2. Energy and fatigue \\
3. Sleep and rest \\
9. Mobility \\
10. Activities of daily living \\
11. Dependence on medicinal substances and medical aids \\
12. Work capacity \\
4. Positive feelings \\
5. Thinking, learning, memory and concentration \\
6. Self-esteem \\
7. Bodily image and appearance \\
8. Negative feelings \\
24. Spirituality/religion/personal beliefs \\
13. Personal relationships \\
14. Social support \\
15. Sexual activity \\
16. Freedom, physical safety and security \\
17. Home environment \\
18. Financial resources \\
19. Health and social care: accessibility and quality \\
20. Opportunities for acquiring new information and skills \\
21. Participation in and opportunities for recreation/leisure activities \\
22. Physical environment (pollution/noise/traffic/climate) \\
23. Transport \\
4. Environment
\end{tabular}


team; the participant should attend the computer lab on the date of the retest application defined by the WHOQOL-Bref/ Libras team. Thus, the two meetings were held on-site, in a computer lab that had laptops and the internet. The test and retest were performed with an interval of 15 days.

In the first meeting there was a presentation of the project, its objectives and importance, presentation of the software and explanations about its use and handling. Participants then received the user code and password for this step and answered the questionnaires. After 2 weeks, the second meeting allowed the same deaf, with new user code and password, to participate, accessing and answering the software questionnaires. All participants completed the Informed Consent Form.

\section{Data analysis}

To perform the data analysis, the classic psychometric tests that assess the reliability and validity of the instrument were used. The Shapiro-Wilk test was used to assess the normality of the distribution of scales and subscales.

The internal consistency of the scales and subscales was analyzed by the Cronbach's alpha coefficient. The temporal stability of the instrument was performed with the 52 participants who completed the retest using the intraclass correlation coefficient (ICC, mean of measures, with two random effects) and Pearson correlation.

To study the discriminant validity of the instrument, we used the question: "Do you have any disease? (Dichotomous answer Yes/No)." Whereby individuals were classified as sick and healthy. In addition to this variable, the following demographic variables were chosen to study discriminant validity: age (composed of two categories: 18 to 40 years and 41 to 65 years); education (consisting of two categories: 0 to 11 years of schooling and 12 or more years of schooling); sex (male and female); and income (consisting of two categories: up to $\mathrm{R} \$ 800$ and above $\mathrm{R} \$ 800$ ). For comparisons of means (or distributions), the effect size and its respective confidence interval were calculated.

Concerning the education, the surveyed population was divided into two groups. The group of deaf people with schooling up to high school (basic learning cycle) would be those who have up to 11 years of study, and the other group that would have entered higher education, with 12 years of study or more. This grouping decision is based on data from the National Continuous Household Sample Survey (PNAD, in Portuguese) of 2018, carried out by the Brazilian Institute of Geography and Statistics (IBGE).

Confirmatory factor analysis (CFA) was used to test the structure of WHOQOL-Bref/Libras, according to Skevington [20]. The CFA was performed using the SPSS AMO Analysis of Moments Structures (IBM SPSS) program [21]. It was performed using the 26 items as observed variables of the model. The four domains (Physical health, Psychological, Social relation ships and Environment) were latent variables. To evaluate the model fit, three index were used: (i) the Chi-square index on degrees of freedom (CMIN/DF) to obtain a fit value to the model less sensitive to the sample size; (ii) the normalized adjustment index (NFI), assuming that the measured variables are completely independent; and (iii) the comparative fit index (CFI), which considers the complexity of the model and the Root Mean Square Error of Approximation (RMSEA) and more adequately represents how well a model fits the population [22].

For data analysis, the following programs were used: R3.2.2 for calculation of effect sizes and their confidence intervals [23] with the packages effsize [24], orddom [25], and MBESS [26], and AMOS [21] for confirmatory factor analysis and SPSS [27] for the other analyses.

\section{Results}

The results obtained in the application of WHOQOL-Bref/ Libras are presented in Tables 2, 3, 4, 5, 6, 7, 8, and 9.

The sample consisted of 311 deaf communicating through Libras, from the five Brazilian regions, including two states in the Northern region: Pará and Tocantins (3.9\%); three from the Northeast: Bahia, Ceará and Rio Grande do Norte (1.2\%); three from the Southeast: Rio de Janeiro, São Paulo and Minas Gerais (8.7\%); three from the South: Paraná, Rio Grande do Sul and Santa Catarina (10.6\%) four from the Midwest: Goiás, Mato Grosso, Mato Grosso do Sul and Distrito Federal (75.5\%).

It was observed that there was a preponderance of female respondents, single marital status, no children, education level of 12 years or over, active working life, wage income over $\mathrm{R} \$ 800.00$, with an average age of 36.6 years (SD 12.34). Of the total, 259 (83.28\%) deaf people participated at a distance, while $52(16.72 \%)$ of them deaf participated in the Computer Lab, and $100 \%$ used the internet. The sociodemographic profile of the studied population can be observed in Table 2. The scores for the entire group, including the group that participated in the test and retest, are shown in the Table 3.

\section{Internal consistency}

The values achieved by the Cronbach's alpha are presented in Table 3 and are between 0.450 and 0.873 . The overall value is considered satisfactory, but individually, the social domain was below the expected value (0.450) [28]. 
Table 2 Sociodemographic profile of the deaf population communicating by Libras $(n=311)$

\begin{tabular}{ll}
\hline Characteristic & $n(\%)$ \\
\hline Age (years) & $36.66(12.3)^{\mathrm{a}}$ \\
18(min)-64(máx.) & \\
Sex & $140(45.0)$ \\
Masculine & $171(55.0)$ \\
Feminine & \\
Marital status & $131(42.1)$ \\
Married & $180(57.9)$ \\
Not married & \\
Occupational status & $104(33.4)$ \\
Unemployed & $207(66.6)$ \\
Employed & \\
Monthly income & $133(42.8)$ \\
$<$ R $\$ 00.00$ & $178(57.2)$ \\
$>$ R $\$ 800.00$ & \\
Number of children & $179(57.6)$ \\
Childless & $132(42.4)$ \\
With child & \\
Years of school & $130(41.8)$ \\
0 to 11 years & $181(58.2)$ \\
12 Years or more & \\
Health condition ${ }^{\mathrm{b}}$ & $116(37.3)$ \\
Sick & $195(62.7)$ \\
Healthy &
\end{tabular}

${ }^{a}$ Average (SD)

${ }^{\mathrm{b}}$ Self-declared health condition

Table 3 WHOQOL-Bref/Libras Domain scores of the deaf sample population considering mean and standard deviation (SD)

\begin{tabular}{lclc}
\hline Domain/factor $^{\mathrm{a}}$ & Average (SD) & Cronbach's alpha & $\begin{array}{c}\text { Number } \\
\text { of items }\end{array}$ \\
\hline Physical health & $66.00(13.66)$ & 0.641 & 7 \\
Psychological & $70.62(13.54)$ & 0.705 & 6 \\
$\begin{array}{l}\text { Social relation- } \\
\text { ships }\end{array}$ & $70.85(15.26)$ & 0.450 & 3 \\
Environment & $60.69(13.15)$ & 0.710 & 8 \\
Overall-Bref & $\begin{array}{c}75.00(62,50 ; \\
87,50)^{\mathrm{b}}\end{array}$ & 0.873 & 26 \\
& & &
\end{tabular}

Internal consistency of WHOQOL-Bref/Libras domains, as measured by Cronbach's alpha, of deaf sample population using sign Libras ( $n$ $=311$ )

${ }^{a}$ WHOQOL-Bref/Libras: factors are consistent with those found in the original project (global sample) [13]

${ }^{\mathrm{b}}$ Median (1st and 3rd quartiles)

\section{Temporal stability}

The test and retest, with 52 participants, was used to verify the temporal stability of the instrument, which took place within 15 days. The domain averages obtained before and after indicate temporal stability. Table 4 shows the correlation coefficients between test and retest data. It is noticed that these coefficients presented high values (above 0.7 ), which is highly significant and satisfactory. It can be observed that the physical health, psychological and environment domains present values of correlation coefficient that are close. In addition, as in the global sample (Table 3), the group that participated in the test and retest obtained similar scores for the Psychological and Social relationships domains.

\section{Construct validity}

The validity of the WHOQOL-Bref/Libras construct was analyzed in relation to health status. For this, the mean scores of each domain and Overall-Bref between healthy and sick groups were compared. The mean scores for the Physical, Psychological, and Social domains were significantly higher in healthy deaf individuals than in sick deaf individuals (Table 5).

\section{Discriminant validity}

\section{In relation to age}

The younger group (aged up to 40 years) did not have different mean scores than the older group (aged over 40 years) in relation to any of the WHOQOL-Libras domains (Table 6).

\section{In relation to years of school}

The years of study were associated with higher average scores in all domains of WHOQOL, that is, deaf individuals with 12 years of schooling or more presented higher scores in the Physical and Psychological domains than individuals with 11 or less years of schooling (Table 7).

In the variable "years of school", the significance level $p<0.05$ was evidenced for the Psychological Domain and the significance level $p \leq 0.001$ for the Physical, Social, Environment Domains. 
Table 4 Comparison between WHOQOL-Bref/Libras domain scores of the deaf sample population $(n=52)$

\begin{tabular}{|c|c|c|c|c|c|}
\hline \multirow[t]{2}{*}{ Domain/factor } & \multicolumn{2}{|l|}{ Average (SD) } & \multirow[t]{2}{*}{$d(\mathrm{CI} 95 \%)$} & \multirow[t]{2}{*}{$\mathrm{ICC}^{\mathrm{a}}(\mathrm{CI} 95 \%)$} & \multirow{2}{*}{$\begin{array}{l}\text { Correlation } \\
\text { coefficient }^{\mathrm{a}}\end{array}$} \\
\hline & Test & Retest & & & \\
\hline Physical health & $64.42(13,59)$ & $64.49(13,70)$ & -0.009 & $0.830^{\mathrm{b}}(-2.30 ; 2.16)$ & $0.827^{\mathrm{e}}$ \\
\hline Psychological & $69.87(13.55)$ & $69.07(15.00)$ & 0.097 & $0.835^{\mathrm{b}}(-1.49 ; 3.09)$ & $0.838^{\mathrm{e}}$ \\
\hline Social relationships & $72.11(14.08)$ & $69.87(15.93)$ & 0.192 & $0.694^{\mathrm{b}}(1.00 ; 5.49)$ & $0.703^{\mathrm{e}}$ \\
\hline Environment & $61.60(12.07)$ & $62.62(13.08)$ & -0.143 & $0.839^{\mathrm{b}}(-3.00 ; 0.96)$ & $0.842^{\mathrm{e}}$ \\
\hline Overall-Bref & $75.00(62.50 ; 87.50)^{\mathrm{c}}$ & $75.00(62.50 ; 87.50)^{\mathrm{c}}$ & $-0.038^{\mathrm{d}}$ & $0.774(-0.20 ; 0.13)$ & $0.773^{\mathrm{e}}$ \\
\hline
\end{tabular}

${ }^{a}$ WHOQOL-Bref/Libras: factors are consistent with those found in the original project (global sample) [13]

${ }^{\mathrm{b}} p<0.05 ; p \leq 0.001 . d=$ effect size

${ }^{\mathrm{c}}$ Median (1st and 3rd quartiles)

${ }^{\mathrm{d}}$ Variable without normal distribution with $d$ values calculated by $d$ Cliff. The others with $d$ value calculated by Cohen

${ }^{\mathrm{e}} p \leq 0.001$

Table 5 WHOQOL-Bref/Libras construct validity in relation to health status of deaf sample population using Libras $(N=$ 311)

\begin{tabular}{llll}
\hline Domain/factor & \multicolumn{2}{l}{ Sick } & df $=309$ \\
\cline { 2 - 3 } & No $(n=195)$ & Yes $(n=116)$ & \\
& Average $(\mathrm{SD})$ & Average $(\mathrm{SD})$ & $\mathrm{d}(\mathrm{CI} 95 \%)$ \\
\hline Physical health & $68.99(12.52)$ & $60.96(13.26)$ & $0.627(0.391 ; 0.864)$ \\
Psychological & $72.88(12.42)$ & $66.81(14.49)$ & $0.459(0.224 ; 0.693)$ \\
Social relationships & $73.24(13.85)$ & $66.81(16.66)$ & $0.430(0.196 ; 0.664)$ \\
Environment & $61.81(13.01)$ & $58.80(13.21)$ & $0.229(-0.002 ; 0.461)^{\mathrm{a}}$ \\
Overall-Bref & $75.00(75.00 ; 87.50)^{\mathrm{b}}$ & $75.00(62.50 ; 75.00)^{\mathrm{b}}$ & $-0.286(-0.402 ;-0.161)$ \\
\hline
\end{tabular}

Factors are consistent with those found in the original project (global sample) [13]

${ }^{a}$ Variable without normal distribution with $d$ values calculated by $d$ Cliff. The others with $d$ value calculated by Cohen

${ }^{\mathrm{b}}$ Median (1st and 3rd quartiles)

\begin{tabular}{llll}
\hline Domain/factor & \multicolumn{2}{l}{ Age (years) } & df $=309$ \\
\cline { 2 - 3 } & $18-40(n=205)$ & $41-64(n=106)$ & \\
& Average (SD) & Average (SD) & $d($ CI 95\%) \\
\hline Physical health & $66.88(13.21)$ & $64.28(13.53)$ & $0.195(-0.431 ; 0.042)$ \\
Psychological & $71.46(13.49)$ & $68.98(13.52)$ & $0.184(-0.420 ; 0.053)$ \\
Social relationships & $72.52(15.13)$ & $67.61(15.05)$ & $0.325(-0.563 ;-0.087)$ \\
Environment & $61.17(12.99)$ & $59.75(13.44)$ & $0.107(-0.344 ; 0.129)$ \\
Overall-Bref & $75.00(62.50 ; 87.50)^{\mathrm{a}}$ & $75.00(62.50 ; 75.00)^{\mathrm{a}}$ & $0.097^{\mathrm{b}}(-0.033 ; 0.225)$ \\
\hline
\end{tabular}

Factors are consistent with those found in the original project (global sample) [13]

${ }^{a}$ Median (1st and 3rd quartiles)

${ }^{\text {b}}$ Variable without normal distribution with $d$ values calculated by $d$ Cliff. The others with $d$ value calculated by Cohen

\section{In relation to sex}

Mean WHOQOL domain scores for the sex variable showed statistically significant differences in the Physical Domain (Table 8).

\section{In relation to income}

Regarding the income variable, deaf individuals with monthly income above R $\$ 800.00$ presented higher scores only in the WHOQOL Physical domain (Table 9). 
Table 7 WHOQOL-Bref/ Libras discriminant validity for variable "years of school"
Table 8 WHOQOL-Bref/ Libras discriminant validity for variable "sex"
Table 9 WHOQOL-Bref/ Libras discriminant validity for variable "monthly income"

\begin{tabular}{|c|c|c|c|}
\hline \multirow[t]{3}{*}{ Domain/factor } & \multicolumn{2}{|l|}{ Years of school } & \multirow{3}{*}{$\begin{array}{l}\mathrm{df}=309 \\
d(\mathrm{CI} 95 \%)\end{array}$} \\
\hline & $0-11(n=130))$ & 12 ou more $(n=181$ & \\
\hline & Average (SD) & Average (SD) & \\
\hline Physical health* & $63.02(12.89)$ & $68.13(13.31)$ & $0.389(-0.618 ;-0.160)$ \\
\hline Psychological** & $67.21(13.78)$ & $73.06(12.83)$ & $0.442(-0.672 ;-0.212)$ \\
\hline Social relationships* & $68.26(16.92)$ & $72.69(13.69)$ & $0.293(-0.521 ;-0.065)$ \\
\hline Environment* & $58.87(13.47)$ & $61.99(12.78)$ & $0.239(-0.467 ;-0.012)$ \\
\hline Overall-Bref & $75.00(62.50 ; 87.50)^{\mathrm{a}}$ & $75.00(62,50 ; 87.50)^{\mathrm{a}}$ & $0.052^{\mathrm{b}}(-0.075 ; 0.178)$ \\
\hline
\end{tabular}

Factors are consistent with those found in the original project (global sample) [13]

${ }^{a}$ Median (1st and 3rd quartiles)

${ }^{\mathrm{b}}$ Variable without normal distribution with $d$ values calculated by $d$ Cliff. The others with $d$ value calculated by Cohen. Significance level: ${ }^{*} p<0.05,{ }^{* *} p \leq 0.001$

\begin{tabular}{llll}
\hline Domain/factor & \multicolumn{2}{l}{ Sex } & df $=309$ \\
\cline { 2 - 3 } & Male $(n=140)$ & Female $(n=171)$ & \\
& Average $(\mathrm{SD})$ & Average $(\mathrm{SD})$ & \multirow{2}{*}{$d(\mathrm{CI} 95 \%)$} \\
\hline Physical health & $69.46(12.70)$ & $63.15(13.24)$ & $-0.544(-0.713 ;-0.257)$ \\
Psychological & $72.67(13.57)$ & $68.93(13.30)$ & $-0.262(-0.053 ;-0.053)$ \\
Social relationships & $75.00(66.67 ; 83.33)^{\mathrm{a}}$ & $75.00(66.67 ; 75.00)^{\mathrm{a}}$ & $0.084^{\mathrm{b}}(-0.043 ; 0.209)$ \\
Environment & $61.78(13.92)$ & $59.79(12.44)$ & $-0.151(-0.376 ; 0.073)$ \\
Overall-Bref & $75.00(75.0 ; 87.5)^{\mathrm{a}}$ & $75.00(62.50 ; 87.50)^{\mathrm{a}}$ & $0.118^{\mathrm{b}}(-0.007 ; 0.240)$ \\
\hline
\end{tabular}

Factors are consistent with those found in the original project (global sample) [13]

${ }^{a}$ Median (1st and 3rd quartiles)

${ }^{\mathrm{b}}$ Variable without normal distribution with $d$ values calculated by $d$ Cliff. The others with $d$ value calculated by Cohen

\begin{tabular}{lllr}
\hline Domain/factor & \multicolumn{2}{l}{ Monthly income } & $\mathrm{df}=309$ \\
\cline { 2 - 3 } & $\geq \mathrm{R} \$ 800.00(n=178)$ & $<\mathrm{R} \$ 800.00(n=133)$ & \\
& Average $(\mathrm{SD})$ & Average $(\mathrm{SD})$ & $d(\mathrm{CI} 95 \%)$ \\
\hline Physical health & $68.43(12.77)$ & $62.73(13.48)$ & $0.436(0.208 ; 0.665)$ \\
Psychological & $71.70(13.54)$ & $69.17(13.44)$ & $0.187(-0.040 ; 0.414)$ \\
Social relationships & $75.00(66.67 ; 83.33)^{\mathrm{a}}$ & $75.00(66.67 ; 75.00)^{\mathrm{a}}$ & $-0.009^{\mathrm{b}}(-0.136 ; 0.118)$ \\
Environment & $61.90(12.74)$ & $59.07(13.56)$ & $0.216(-0.011 ; 0.443)$ \\
Overall-Bref $(a)$ & $75.00(62.50 ; 87.50)^{\mathrm{a}}$ & $75.00(62.50 ; 87.50)^{\mathrm{a}}$ & $-0.029^{\mathrm{b}}(-0.155 ; 0.098)$
\end{tabular}

Factors are consistent with those found in the original project (global sample) [13]

${ }^{a}$ Median (1st and 3rd quartiles)

${ }^{\mathrm{b}}$ Variable without normal distribution with $d$ values calculated by $d$ Cliff. The others with $d$ value calculated by Cohen
Among two variables observed in Tables 8 and 9, there was statistical significance for Sex and Monthly income. For the significance level $p<0.05$, the variable "sex" showed the Physical health and Psychological domains. For the significance level $p \leq 0.001$ in the variable "monthly income," the Physical health domain was evidenced.

\section{Confirmatory factor analysis}

The CFI compared the hypothetical model, which contains four correlated dimensions, with what occurs when applying the instrument to the sample in question, considering that for this index the appropriate value is 0.9 . A lower than expected $\mathrm{CFI}=0.718$ was found, and values for $\mathrm{CMIN} / \mathrm{DF}$ 


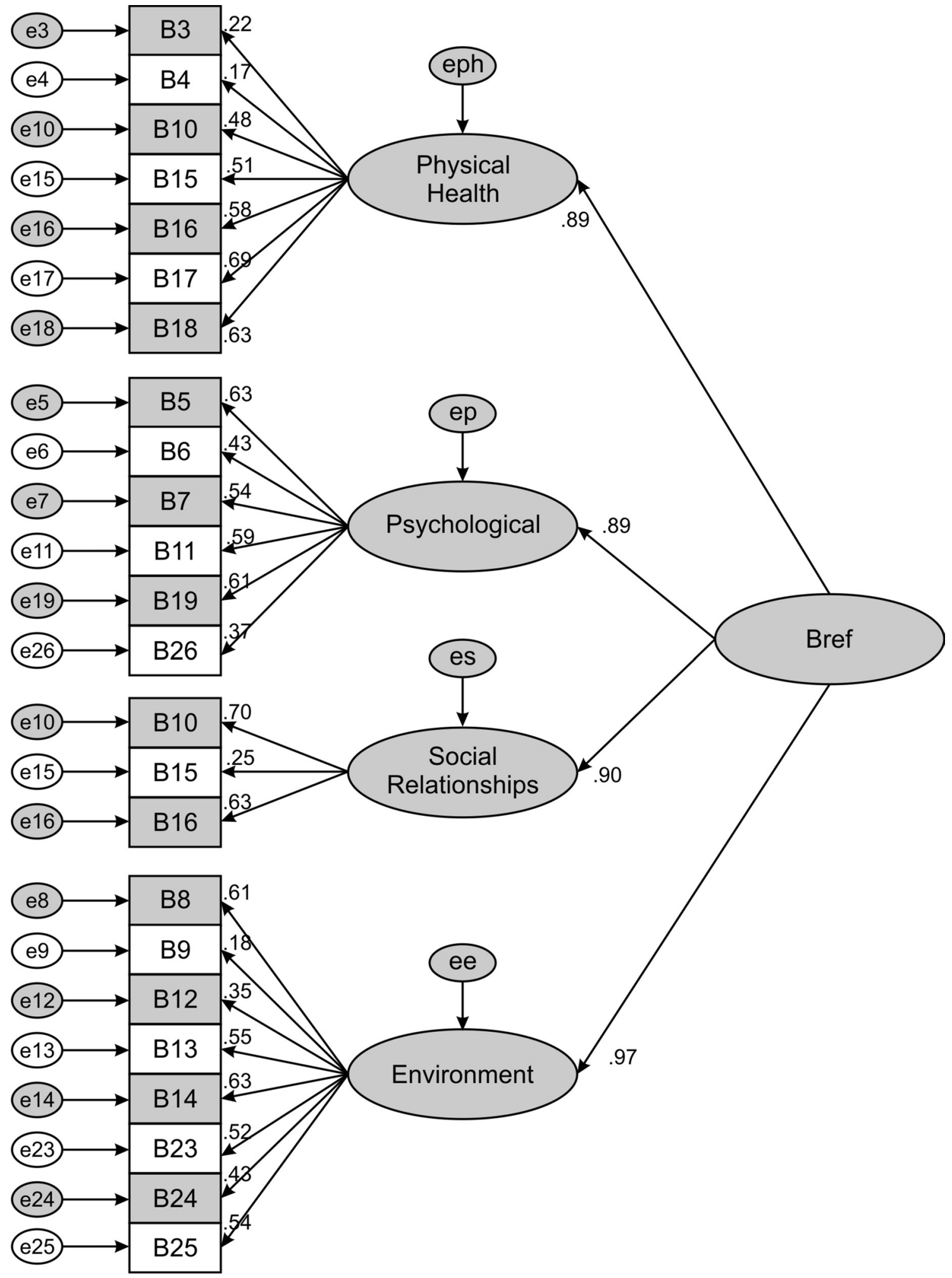

Fig. 1 Confirmatory factor analysis result

$=3.224 ; \mathrm{NFI}=0.642 ; \mathrm{RMSEA}=.085$. Figure 1 represents a factorial model of the items that make up the instrument (observed variables) and their dimensions (latent variables).
It is possible to observe all the standardized effects of each item, for each domain. Most items have a correlation coefficient of 0.40 or greater. 


\section{Discussion}

This study contributes significantly to deaf people's health research because it demonstrates that the WHOQOL-Bref/ Libras version has satisfactory fundamental psychometric characteristics, such as construct validity, discriminant validity, test-retest reliability, and internal consistency. This is particularly important because the official language of Brazilian deaf people is Libras, enabling the application of WHOQOL-Bref in accordance with the values of deaf culture. In addition, having an instrument in Libras allows you to accurately characterize the health inequalities in Deaf population.

Cronbach's alpha coefficient showed satisfactory values for the Physical health, Psychological, Environment, and Overall-Bref domains (sum of 26 items). The Social relationships domain presented an unsatisfactory value (0.450). This can be explained by the fact that the Cronbach's alpha coefficient is a highly sensitive test for size and number of items per factor [29]. The lowest values found in most validation studies of this instrument were in the Social relationships domain. In the study that considers the application of the WHOQOL-Bref instrument in Portuguese and applied in written form, disregarding deaf populations, this coefficient also presented the lowest values of internal consistency (0.69) [30]. A study conducted to evaluate WHOQOL-Bref in a sample of the general population of Sudan also found a low value very close to WHOQOL-Bref/Libras [31].

Regarding Temporal Stability, analyzed in the test-retest reliability by Pearson's Correlation Coefficient and the $t$-Test, the WHOQOL-Bref/Libras presented little variation in the averages between the two moments, with high correlation coefficients between the two measures in all domains. Thus, the analysis concluded that the WHOQOL-Bref/Libras is a stable instrument over time, when the individual's conditions remain approximately the same. The discriminatory capacity of the WHOQOLBref/Libras was verified in the variables age, sex, monthly income, and educational level. In the variable age no significant values were found.

When compared with WHOQOL normative data in a randomized population-based sample in southern Brazil, some similarities were found [32]. As in the population sample, among the deaf there was a better quality of life in all domains in individuals with higher years of schooling. Sick individuals from both the population-based and deaf sample had worse quality of life scores in all domains except the Social relationships domain among the deaf sample. The lower value found in the social domain is due to the fact that Cronbach's alpha is highly sensitive to the number of items. The Social relationships domain has 3 items. Unlike the deaf sample, in the population-based sample, male and Brazilian individuals had better scores in different domains of quality of life [33].

Regarding age, only very young individuals (up to 29 years old) from the population-based sample showed better scores in some WHOQOL domains. The other strata did not present better scores. In the deaf sample, there was no difference in age. As the way to stratify the socioeconomic level in this work was different from the stratification adopted in Cruz et al. [32], the comparison became difficult. In the present study with the deaf sample, socioeconomic status was assessed by monthly income. In the population-based study by Cruz et al., this variable was evaluated by an economic class measure. Deaf people with higher income had higher scores in the Physical domain, being equal in the other domains. When the income criterion was used in the population-based study, there was an association between higher social class and better scores in all WHOQOL domains.

In relation to years of schooling, according to the National Survey by Sample of Continuing Households (PNAD, in Portuguese) of 2018, carried out by the Brazilian Institute of Geography and Statistics (IBGE), 52.6\% of Brazilians aged 25 or over did not complete the study-minimum expected study. The majority, $33.1 \%$, did not even finish elementary school. In this research, $41.8 \%$ of the deaf have up to 11 years of study, and 58.2 have entered higher education, with 12 years of study or more. Regarding quality of life, in the work of Cruz [32], differences were observed in the mean scores between the groups with the lowest educational level (up to 4 years) and the highest (12 years or more) in almost all domains. Higher scores were observed in the group with more years of schooling. In this study, deaf people with 12 years of schooling or more had higher scores in the Physical and Psychological domains than individuals with 11 years or less of schooling.

Confirmatory Factor Analysis was also used to assess the WHOQOL-Bref/Libras construct validity by testing its structure. For NFI and CFI a value of $\geq 0.09$ is expected, GFI above 0.90 and for RMSEA values below 0.08 are considered desirable; values below 0.06 generally indicate a reasonable fit to the model [22]. The CFI compared the hypothetical model that contains four correlated dimensions with what occurs when applying the instrument to the sample in question, considering that for this index the appropriate value is 0.9 . A lower $\mathrm{CFI}=0.718$ was found than desired. While the values for $\mathrm{CMIN} / \mathrm{DF}=3.224$; NFI $=$ 0.642 ; RMSEA $=.085$.

Henning et al. [16] reported that the deaf community group scored lower than the general New Zealand sample on all domains. New Zealand deaf people achieved the highest score in the environment domain, while this domain was the worst assessed by Brazilian deaf people. Communicative barriers prevent access to Brazilian deaf 
people to various socio-cultural activities. The difficulty of having Libras interpreters in access to different social environments explains the lower score in relation to Henning's study. Brazilian deaf people achieved the highest scores in the social and psychological relations domains, while New Zealand deaf people reached the worst score in the social relations domain.

We used the study by Cruz et al. [32] to compare with the data of the present study, since it is the study that offers the greatest possibility of comparison. However, there are important methodological differences between the two studies. First, it is a randomized population-based sample from southern Brazil, while the present study was conducted from a convenience sample. Second, the Cruz study sample is composed exclusively of individuals who speak the Portuguese language and are not deaf, while the present study is exclusively of deaf people who answered a version of WHOQOL in Brazilian Sign Language.

The present study has some limitations. First, it was not possible to use other sign language instruments that would allow us to analyze the convergent validity of WHOQOLBref/Libras with other quality of life measures or any other construct (such as a depression scale). This is due to the pioneering character of this project. Furthermore, making WHOQOL-Bref/Libras available could be an important tool for ambitious project involving a significant portion of the country's deaf population.

Although these analyzes provide good support for the use of WHOQOL-Bref/Libras, further research needs to be performed, as the validation of an instrument is done through the continuous process of improvement. A further step toward a better understanding of the performance of the sign language version of the WHOQOL-Bref in relation to the instrument in Portuguese could be the application in a bilingual sample, which dominates the written modality of Portuguese and Libras.

Acknowledgements Research supported by the Ministério da Saúde/ SCTIE/DECIT, Conselho Nacional de Pesquisa e Desenvolvimento Tecnológico (Grant Number 429235/2016-0), and by the Fundação de Amparo à Pesquisa do Estado de Goiás (FAPEG) (DECIT-SCTIE-MS, Grant 200910267000540).

\section{Compliance with ethical standards}

Conflict of interest The authors declare that they have no conflict of interest.

Ethical approval The Research Ethics Committee of the Federal University of Goiás approved the project in accordance with the Resolutions in force 196/466, of 1996/2012, of the National Health Council and authorized the research through resources and virtual environment [34]. All procedures performed in this work were in accordance with the ethical standards of the institutional research committees and with the 1964 Helsinki declaration and its later amendments or comparable ethical standards. Informed consent was obtained from all individual participants included in the study.

\section{References}

1. Fellinger, J., Holzinger, D., Gerich, J., \& Goldberg, D. (2007). Mental distress and quality of life in the hard of hearing. Acta Psychiatrica Scandinavica, 115(3), 243-245.

2. Padden, Carol, \& Humphries, Tom. (2006). Inside deaf culture. Cambridge: Harvard University Press.

3. Ladd, P. (2003). Understanding deaf culture: In search of deafhood. Clevedon: Multilingual Matters.

4. Skliar, C. (1998). A Surdez: um olhar sobre as diferenças. Editora Mediação: Porto Alegre.

5. Duarte, S. B. R., Chaveiro, N., de Freitas, A. R., Barbosa, M. A., Porto, C. C., \& de Almeida Fleck, M. P. (2013). Aspectos históricos e socioculturais da população surda. Hist. cienc. saudeManguinhos, 20, 1713-1734.

6. Kuenburg, A., Fellinger, P., \& Fellinger, J. (2015). Health care access among deaf people. The Journal of Deaf Studies and Deaf Education, 21(1), 1-10.

7. Martins, K. P., da Costa, T. F., Medeiros, T. M., Fernandes, M. G. M., França, I. S. X. D., \& MacêdoCosta, K. N. F. (2016). Internal structure of family health units: Access for peoplewith disabilities. Ciência \& Saúde Coletiva, 21, 3153-3160.

8. Sheppard, K. (2014). Deaf adults and health care: Giving voice to their stories. Journal of the American Association of Nurse Practitioners, 26(9), 504-510.

9. Castro, S. S., Lefreve, F., Lefreve, A. M. C., \& Cesar, C. L. G. (2011). Accessibility to health services by persons with disabilities. Revista de saude publica. https://doi.org/10.1590/S0034 -89102010005000048.

10. Anderson, M. L., Riker, T., Hakulin, S., Meehan, J., Gagne, K., Higgins, T., et al. (2019). Deaf ACCESS: Adapting consent through community engagement and state-of-the-art simulation. The Journal of Deaf Studies and Deaf Education, 25(1), 115-125.

11. Pendergrass, K. M., Newman, S. D., Jones, E., \& Jenkins, C. H. (2019). Deaf: A concept analysis from a cultural perspective using the wilson method of concept analysis development. Clinical Nursing Research, 28(1), 79-93.

12. Haraldstad, K., Wahl, A., Andenæs, R., Andersen, J. R., Andersen, M. H., Beisland, E., et al. (2019). A systematic review of quality of life research in medicine and health sciences. Quality of Life Research An International Journal of Quality of Life Aspects of Treatment, Care and Rehabilitation: An Official Journal of the International Society of Quality of Life Research, 28(06), 2641-2650.

13. Harper, A., Power, M., Orley, J., Herrman, H., Schofield, H., Murphy, B., et al. (1998). Development of the World Health Organization WHOQOL-BREF Quality of Life Assessment. Psychological Medicine, 28(3), 551-558. 5.

14. Cieza, A., \& Stucki, G. (2005). Content comparison of healthrelated quality of life (hrqol) instruments based on the international classification of functioning, disability and health (icf). Quality of Life Research An International Journal of Quality of Life Aspects of Treatment, Care and Rehabilitation - An Official Journal of the International Society of Quality of Life Research, 14, 1225-1237.

15. Fellinger, J., Holzinger, D., Dobner, U., Gerich, J., Lehner, R., Lenz, G., et al. (2005). An innovative and reliable way to measure health-related quality of life and mental distress in the deaf community. Social Psychiatry and Psychiatric Epidemiology, 40, $245-50$. 
16. Henning, M. A., Krägeloh, C. U., Sameshima, S., Shepherd, D., Shepherd, G., \& Billington, R. (2011). Access to New Zealand sign language interpreters and quality of life for the deaf: A pilot study. Disability and Rehabilitation, 33(25-26), 2559-2566.

17. Jaiyeola, M. T., \& Adeyemo, A. A. (2018). Quality of life of deaf and hard of hearing students in ibadan metropolis, Nigeria. PLoS ONE, 13(1), 1-11.

18. Chaveiro, N., Duarte, S. B. R., Freitas, A. R. D., Barbosa, M. A., Porto, C. C., \& Almeida, F. M. P. D. (2013). Instruments in Brazilian Sign Language for assessing the quality of life of the deaf population. Revista de Saúde Pública, 47, 616-623.

19. Chaveiro, N., Rodrigues, C. L., Duarte, S. B., Rodrigues, de Oliveira Garcia, R., Ribeiro de Freitas, A., Rodrigues de Oliveira, L., Rocha Gomes da Silva, K., Porto C. C.(2018). A tool to evaluate the quality of life of deaf people using WHOQOL instruments. In 2018 IEEE international conference on systems, man, and cybernetics (SMC) (pp. 3871-3876).

20. Skevington, S. M., Lotfy, M., \& O'Connell, K. A. (2004). The World Health Organisation's WHOQOL-BREF Quality of Life Assessment: Psychometric properties and results of the international field trial-A report from the WHOQOL Group. Quality of Life Research, 13(2), 299-310.

21. Barnidge, M., Homero, G. (2017). Amos (Software) (p. 11).

22. Thompson, B. (2004). Exploratory and confirmatory factor analysis: Understanding concepts and applications. Structural Equation Modeling-a Multidisciplinary Journal_STRUCT EQU MODELING, 15, 01.

23. R Development Core Team. (2019). R: A Language and Environment for Statistical Computing.

24. Torchiano, M. (2016). Effsize: Efficient effect size computation. $\mathrm{R}$ package version 0.6.1.

25. Rogmann, J. J. (2013). Ordinal dominance statistics (orddom): An $\mathrm{R}$ Project for Statistical Computing package to compute ordinal, nonparametric alternatives to mean comparison (Version 3.1)

26. Kelley, K. (2017). Confidence intervals for standardized effect sizes: Theory, application, and implementation. $j$-J-STAT-SOFT, $20(8), 1-24$.
27. George, D., \& Mallery, P. (2016). IBM SPSS statistics 23 step by step: A simple guide and reference (14th ed.). New York: Routledge.

28. Verma, J. P., \& Abdel-Salam, A.-S. (2019). Testing statistical assumptions in research. New York: Wiley.

29. Streiner, D. L. (2003). Starting at the beginning: An introduction to coefficient alpha and internal consistency. Journal of Personality Assessment, 80(1), 99-103.

30. Fleck, M., Louzada, S., Xavier, M., Chachamovich, E., Vieira, G., Santos, L., et al. (2000). Application of the Portuguese version of the abbreviated instrument of quality life WHOQOL-Bref. Revista de Saúde Pública, 34(2), 178-183.

31. Ohaeri, J. U., Awadalla, A., El-Abassi, A.-H., \& Jacob, A. (2007). Confirmatory factor analytical study of the WHOQOL-Bref: Experience with sudanese general population and psychiatric samples. BMC Medical Research Methodology, 7, 37.

32. Cruz, L., Polanczyk, C., Camey, S., Hoffmann, J., \& Fleck, M. (2011). Quality of life in Brazil: Normative values for the WHOQOL-Bref in a southern general population sample. Quality of Life Research: An International Journal of Quality of Life Aspects of Treatment, Care and Rehabilitation, 20, 1123-9.

33. Fleck, M. P. D. A., Leal, O. F., Louzada, S., Xavier, M., Chachamovich, E., Vieira, G., et al. (1999). Development of the Portuguese version of the OMS evaluation instrument of quality of life. Brazilian Journal of Psychiatry, 21, 19-28.

34. BRAZIL. MINISTRY OF HEALTH. Resolution 196/96 of the National Health Council / MS on Guidelines and Regulatory Standards for Research involving humans. Official Gazette, October 10, 1996.

Publisher's Note Springer Nature remains neutral with regard to jurisdictional claims in published maps and institutional affiliations. 